\title{
Una aproximación a las emociones en los equipos deportivos An approach to emotions in sports teams
}

\author{
Marta Eulalia Blanco García \\ Universidad Complutense de Madrid (España)
}

\begin{abstract}
Resumen. Este trabajo se adentra en la convivencia de equipos deportivos pertenecientes a disciplinas que implican contacto con el equipo rival en su práctica, a partir del análisis empírico procedente de 30 entrevistas en profundidad con entrenadores / as y deportistas de equipos de la Comunidad de Madrid de las disciplinas de fútbol, baloncesto y rugby. En un acercamiento feminista desde la sociología del deporte, se señalan las formas de organización de los equipos deportivos, visibilizando las estrictas jerarquías y sistemas de disciplinamiento normalizados, incidiendo en ciertas prácticas exacerbadas que pueden llegar a justificarse en el contexto. A partir de aquí, se realiza un análisis a través de las especiales sensibilidades del deporte, incidiendo en el estudio de las emociones y las dinámicas afectivas en estos equipos deportivos, reflexionando acerca de su misión como sustento de sistemas que perpetúan fragilidades que darán pie a ciertas vulnerabilidades en el espacio, especialmente hacia las mujeres.
\end{abstract}

Palabras clave: deporte, equipo, género, poder, emociones.

\begin{abstract}
This work analyze the coexistence of sports teams of disciplines that involve contact with the rival team in their practice, based on the empirical analysis from 30 interviews with coaches and athletes of teams from the Community of Madrid of the soccer, basketball and rugby disciplines. In a feminist approach from the sociology of sport, the forms of organization of sports teams are pointed out, making visible the strict hierarchies and normalized disciplinary systems, influencing certain exacerbated practices that can be justified in the context. From here, an analysis is carried out through the special sensitivities of sport that affects the study of emotions and affective dynamics in these sports teams, reflecting on their mission as support for systems that perpetuate fragility that will give rise to certain vulnerabilities in space, especially against women.
\end{abstract}

Keywords: sport, teams, gender, power, emotions.

\section{Introducción}

Practicar deporte siempre ha significado mucho más que realizar una actividad física regulada, competir y/ o superar objetivos colectivos o individuales. El deporte es rutina, hábito, motivación, pero también desconexión, relajación, encuentro y convivencia, e implica una expresión emocional característica (Elias, 1986; Sonlleva, Martínez \& Mon-jas, 2018) que influye en el día a día de quienes lo realizan, creando experiencias intensas (De Lauretis, 1992) que las/os acompañarán a lo largo de su trayectoria deportiva y, en definitiva, su vida. Todavía más, si nos referimos a equipos deportivos, estas emociones, sensibilidades y afectividades se volverán especialmente sensibles en su convivencia, siendo parte imprescindible de sus objetivos y metas, pero también de sus interpretaciones individuales. En esta línea, la atención a las emociones desde la sociología del deporte se hace urgente para entender sus rea-

Fecha recepción: 15-10-21. Fecha de aceptación: 06-12-21

Marta Eulalia Blanco García

martaebl@ucm.es lidades cotidianas (Bermúdez Torres y Saenz-López, 2019). Bericat Alastuey (2020) afirmaba que el estudio de las emociones resultará imprescindible a la hora de estudiar y comprender sociológicamente las intercomunicaciones humanas. Añado a ello que, hablando de los equipos deportivos, nos adentramos en un espacio de especial sensibilidad, con una gestión de las emociones muy característica, donde necesitamos entender no sólo cómo se gestionan estas emociones, sino además cuál es el papel de los diferentes agentes implicados y sus repercusiones; es decir, cómo se expresan e interpretan estas explosiones emocionales especialmente sensibles, así como sus dinámicas afectivas, sus circuitos y los vínculos que se conforman en unos equipos deportivos que se erigen como grupos de personas no elegidos que demandan importantes exigencias emocionales a sus integrantes (Blanco García, 2021).

Se propone en este artículo un breve acercamiento feminista y ordinario a estas tensiones y juegos emocionales y afectivos en equipos deportivos de disciplinas que además implican contacto con el equipo rival en su práctica ${ }^{1}$. Esto, para visibilizar las formas de gestión de estos grupos, atendiendo a la construcción idea- 
lizada de ciertos valores deportivos y señalando puntos conflictivos en sus formas de organización, potenciando una autoridad y poder concentrado en una figura masculinizada de entrenador/a y, definitivamente, pudiendo derivar en la perpetuación de espacios vulnerables, especialmente en los equipos femeninos. Como se ha mencionado hace un instante, a menudo las aproximaciones sociológicas a estas emociones en el deporte se limitan a hacer una valoración de resultados competitivos de deportistas y equipos, pero ¿qué descubriría un estudio de estas emociones, sensibilidades y dinámicas afectivas enfocado simplemente en entender sus realidades, sin tener en cuenta su competitividad? ¿Qué pasa si además nos acercamos a ello teniendo en cuenta esta comprensión de las performatividades de género que atraviesan su convivencia? Actualmente resultará incompleto hacer una investigación sociológica del deporte sin tener en cuenta cómo el género influye en sus cotidianidades, con propuestas actuales que amplíen las posibilidades y demuestren cómo muchas de sus consideraciones asentadas resultan problemáticas para un deporte que necesita adaptarse a las circunstancias actuales, haciendo una promoción (real) de muchos de los valores que pretende difundir en sus buenas prácticas. Por último, resulta además urgente cuestionar las bases de estas formas de organización de los equipos deportivos, reflexionando acerca de las tensiones producidas en sus exposiciones de poder y autoridad, dando pie a posibles conductas y prácticas exacerbadas.

\section{Metodología}

El objeto de este trabajo es reflexionar acerca de las dinámicas afectivas en los equipos deportivos, profundizando a través de ellas en sus estructuras y sistemas de organización. Esta es una de las líneas de investigación tratadas en un estudio que ha abordado las dinámicas sexoafectivas en equipos deportivos federados en la Comunidad de Madrid, en tres disciplinas deportivas que implican contacto en su práctica (fútbol, baloncesto y rugby). Todo ello, mediante técnicas de investigación cualitativa, en concreto entrevistas en profundidad ${ }^{2}$.

\section{Participantes}

El diseño muestral incluye un total de 30 entrevistas en profundidad, 15 con deportistas y 15 con entrenadores/as. La investigación se ha reducido a equipos de la Comunidad de Madrid de las disciplinas de fútbol, baloncesto y rugby que se encuentran inscritos en ligas federadas de la propia comunidad o de federaciones na- cionales. Esto, debido al principal interés que suscita la comparación de tres disciplinas que, compartiendo las dos características básicas del estudio: ser deportes de equipo e implicar contacto con el equipo rival en su práctica (lo que tendrá mucho que decir también sobre estereotipos de género y la presencia y participación de mujeres), presentan diferencias en su recorrido y estado actual (en cuanto al número de licencias, promoción y presencia en medios de comunicación, participación, etc.), pero también en sus reglamentos, cotidianidades y convivencia. Dicho esto, los factores que han estructurado el diseño de la muestra han sido el género, la edad y la dedicación deportiva (deportista-cuerpo técnico), además se ha controlado la clase social, siendo todas las entrevistas realizadas a personas de clase social media. No se ha tenido en cuenta el nivel competitivo de los equipos en los que participan las personas entrevistadas por una razón principal: la precariedad del contexto deportivo que afecta especialmente a las mujeres y el deporte femenino. Pues, en este estudio se han mostrado similares dinámicas relatadas por jugadoras que representan selecciones nacionales y aquellas que consideran su práctica deportiva en el equipo sólo un hobby (necesidad de compaginar el deporte con otra(s) actividad(es) económica(s), precariedad en los horarios de entrenamientos y viajes para partidos y torneos, ausencia de contrato laboral, escasez de respaldo y apoyo por parte de clubes y federaciones, etc.) (tabla 1).

\begin{tabular}{|c|c|c|}
\hline \multirow{2}{*}{ Factores } & \multicolumn{2}{|c|}{ Rol deportivo } \\
\hline & Entrenador/a & Deportista \\
\hline Disciplina deportiva & Baloncesto, fútbol y rugby & Baloncesto, fútbol y rugby \\
\hline Género & $\begin{array}{l}\text { Mujeres y hombres, con mayor } \\
\text { incidencia de hombres. }\end{array}$ & $\begin{array}{l}\text { Mujeres y hombres, con mayor } \\
\text { incidencia de mujeres. }\end{array}$ \\
\hline Edad & $\begin{array}{l}\text { Desde los } 18 \text { a los } 40 \text { años, buscando } \\
\text { más incidencia en los hombres desde } \\
\text { los } 28 \text { a los } 40 \text { años. }\end{array}$ & $\begin{array}{l}\text { Desde los } 17 \text { a los } 32 \text { años, buscando } \\
\text { más incidencia en las mujeres de } 17-18 \\
\text { años y } 28-32 \text { años. }\end{array}$ \\
\hline Clase social & $\begin{array}{l}\text { Clase socioeconómica media } \\
\text { (mínimo de Formación Profesional } \\
\text { de Grado Superior o Estudios } \\
\text { Universitarios, cursando o } \\
\text { terminados). }\end{array}$ & $\begin{array}{c}\text { Clase socioeconómica media (mínimo } \\
\text { de Estudios de Bachillerato en el caso } \\
\text { de los/as deportistas menores de edad, } \\
\text { Formación Profesional de Grado } \\
\text { Superior o Estudios Universitarios, } \\
\text { cursando o terminados en los/as } \\
\text { deportistas mayores de edad). }\end{array}$ \\
\hline
\end{tabular}

En cuanto a la edad, se establecen para los y las deportistas cuatro grupos, entre los 17 y los 32 años de edad y para entrenadores/as seis grupos, entre los $17 \mathrm{y}$ los 40 años de edad. Las entrevistas se han distribuido tal y como se muestra en la Tabla 2.

Por último, tal y como puede observarse, existe especial intención de focalizar el estudio con jugadoras jóvenes, dando cierta importancia a la comparación con aquellas más veteranas también, y entrenadores en el final de la etapa joven e inicio y asentamiento de la edad adulta, cuando suelen asentarse en sus roles deportivos 
Tabla 2

Distribución de entrevistas

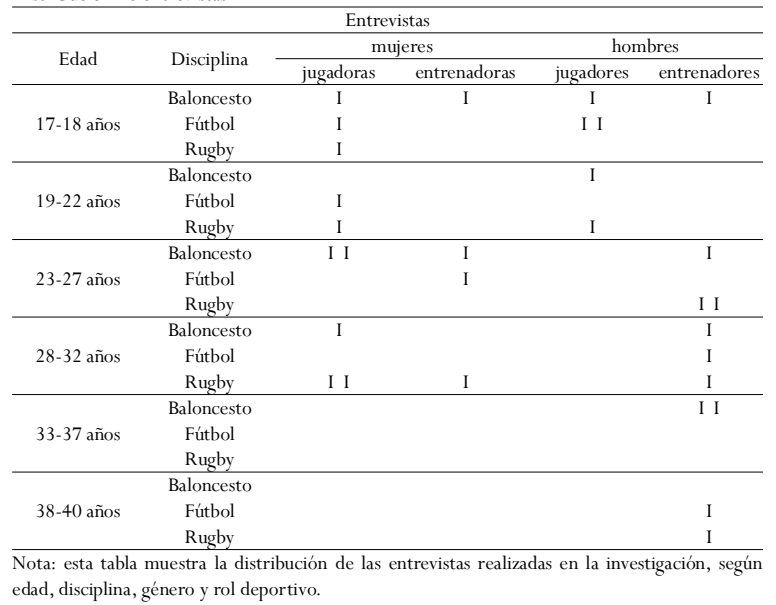

y dar el salto al entrenamiento de equipos jóvenes y adultos. Esto, porque aunque este artículo se centra, como he mencionado anteriormente, en un breve capítulo de la investigación que describe las expresiones emocionales en estos equipos y disciplinas deportivas, el conjunto del estudio analiza dinámicas sexo-afectivas en los equipos deportivos, concluyendo la vulnerabilidad de ciertos espacios en cuanto a la normalización de prácticas de acoso sexual, especialmente en jugadoras jóvenes. En esta línea, tal y como revelan cada vez más estudios (entre los que destaco en este momento: Brackenridge, 2008; Fasting et al., 2003), las jugadoras, sobre todo jóvenes pero no sólo, serán especialmente vulnerables a ciertas situaciones en el ámbito deportivo, relacionadas con estos conflictos en la gestión emocional, así como sufrir acoso sexual por parte de hombres en figuras de poder. Por ello, toman especial interés sus discursos.

\section{Instrumentos de investigación}

La principal herramienta de investigación utilizada ha sido la entrevista en profundidad, debido a la intención de producir información cualitativa que de voz a los y las protagonistas del contexto deportivo, entendiendo las razones y perspectivas más profundas a través de sus discursos (Alonso, 1998). De hecho, tal y como afirma Vicente Olmo (2015), lo realmente interesante de esta herramienta de investigación cualitativa es su distinción de técnicas más tradicionales, centradas principalmente en una recogida de datos cuantitativos (Alonso, 1994). Se buscan pues, nuevas formas de visibilizar los discursos lingüísticos, como eco de relatos, experiencia y/o estilos de vida (Alonso, 1994: 237). Por último, estas individualidades mostradas en las entrevistas, reflejan modelos y referentes culturales en los que las personas conviven diariamente (Finkel et. al.
2008); en este caso, el deporte, sus identificaciones como deportistas y entrenadores/as y los equipos deportivos que construyen, siempre mediados por los estudios de las emociones.

Todas las entrevistas han seguido un guion similar, con escasas puntualizaciones según el rol de la persona de deportista o entrenador/a. Es decir, e confirma un guion con cuatro bloques. En el primero se plantea un acercamiento a la relación actual con el deporte, teniendo en cuenta la formación, el equipo, nivel de competición y dedicaciones actuales. Se hace referencia además a la visión de la trayectoria deportiva a lo largo de la vida. En un segundo bloque se plantean cuestiones de género en el deporte, diferenciando ámbitos personal, familiar e institucional. En un tercer bloque aparece la organización de los equipos deportivos, teniendo en cuenta la jerarquización, la percepción de la autoridad y el poder, así como las formas de comunicación dentro de los equipos, introduciendo por fin la convivencia y las expresiones emocionales en el propio contexto deportivo. Aparece así un cuarto bloque que trata definitivamente las emociones, afectividades y vínculos en los equipos, dirigiéndose hacia las narrativas amorosas y la aparición de ciertos espacios de vulnerabilidad.

\section{Procedimiento de investigación y análisis de datos}

El trabajo de campo se realizó de la siguiente forma (tabla 3)

Tabla 3.

\begin{tabular}{lcc} 
Organización de las oleadas de trabajo de campo & Mujeres & Hombres \\
\hline $1^{\text {a }}$ Oleada campo & $\begin{array}{c}\text { Jugadoras de baloncesto } \\
\text { Jugadoras de rugby } \\
\text { Entrenadora de fútbol }\end{array}$ & Entrenadores de baloncesto \\
\hline $2^{\text {a } \text { Oleada campo }}$ & $\begin{array}{c}\text { Entrenadoras de baloncesto } \\
\text { Entrenadora de rugby }\end{array}$ & $\begin{array}{c}\text { Jugadores de baloncesto } \\
\text { Jugadores de fútbol } \\
\text { Entrenadores de rugby }\end{array}$ \\
\hline $3^{\text {a } \text { Oleada campo }}$ & Jugadoras de fútbol & $\begin{array}{c}\text { Entrenadores de fútbol } \\
\text { Jugador de rugby }\end{array}$ \\
\hline $\begin{array}{l}\text { Nota: esta tabla muestra el procedimiento de investigación llevado a cabo, refiriendo las tres } \\
\text { oleadas de campo realizadas. }\end{array}$ &
\end{tabular}

En la primera oleada se buscó una entrada directa en los terrenos que, según las hipótesis, parecían más vulnerables. Por ello, se escogió el baloncesto: jugadoras y entrenadores de todas las edades. Además, se entrevistó también a las jugadoras de rugby, empezando por las más jóvenes, en contraposición directa entre esos escenarios del rugby que podrían presentar superficialmente otras formas de gestión emocional, debido a sus propias reglas y formas de organización. Una de las entrevistas de una jugadora de rugby cerró la primera oleada. Además, se entrevistó a una entrenadora de fútbol, pues su perfil era muy interesante para ini- 
ciar la investigación, debido a su posición actual en el fútbol español.

El objetivo principal de la segunda oleada era cerrar el estudio del baloncesto, con las entrevistas de las entrenadoras, e introducirse en el fútbol y el rugby, dejando la última oleada simplemente para discursos que resultaran interesantes en puntos concretos. Sin embargo, con el fútbol ha persistido un problema de participación en todos sus roles y franjas de edad. En esta disciplina se llegaron a cancelar hasta 4 entrevistas incluso minutos antes de la hora acordada, tanto por parte de jugadoras como de entrenadores. Esto conllevó una adaptación de las oleadas de trabajo de campo y ha provocado que la participación del fútbol haya sido menor en la investigación, al no conseguir reponerse tampoco en la tercera oleada.

Por último, la tercera oleada introdujo por fin la participación de los entrenadores y una jugadora de fútbol. Además, se determinó zanjar el estudio del rugby con una última entrevista a un jugador, debido a la relevancia de comparar ciertos aspectos relacionados con el liderazgo, la autoridad y la gestión de los grupos en esta y las tres disciplinas deportivas, así como pudiendo realizar comparaciones con perspectiva de género.

En cuanto al análisis de datos, se realizó un estudio de las entrevistas mediante el análisis del discurso. Además, es importante destacar la importante influencia que ha tenido en la propia investigación, tanto en la realización de las entrevistas como el propio análisis de las mismas, la presencia activa en el contexto deportivo. Es decir, desde el cuerpo situado propuesto por Haraway (1988) y la antropología encarnada (Esteban, 2013), se ha podido realizar un acercamiento especialmente cercano y comprensivo, sobre todo con jugadoras y entrenadoras, describiendo esas experiencias intensas (De Lauretis, 1992) que sólo pueden contarse desde los cuerpos.

\section{Resultados del estudio y discusión}

La normalización de las discriminaciones de género en los equipos deportivos

Que el deporte ha sido desde sus orígenes un ámbito muy masculinizado ya ha sido remarcado en numerosas ocasiones (Díez Mintegui, 2006). De la misma forma, se asume que todavía hoy resulta un espacio perfecto para la exaltación de feminidades y masculinidades tradicionales (Pfister, 2010). Ahora bien, a veces caemos en el error de limitar esta masculinización de su recorrido a las prohibiciones explícitas de la práctica deportiva femenina que han sucedido hasta no hace demasiado tiempo ${ }^{3}$, sin tener en cuenta las evidentes discriminaciones y desigualdades a las que seguimos asistiendo. Primeramente, la progresiva adaptación de las mujeres y el deporte femenino se mantuvo mucho tiempo sujeta a prescripciones médicas que consideraban específicamente su misión reproductiva (García García, 2015) ${ }^{4}$, avanzando hacia una mayor libertad en sus dedicaciones deportivas, pero igualmente ligada a estereotipos de género que de la misma forma siguen condicionando estas actividades (Fausto-Sterling, 2000). $\mathrm{Y}$ es que, cabe preguntarnos si realmente han abandonado las prácticas deportivas de las mujeres consideraciones discriminatorias justificadas desde su biología hasta los propios estereotipos que se dirigen supuestamente sus intereses. Es decir, que hoy en día todavía encontremos limitaciones y juicios sociales ligados a la musculación de los cuerpos femeninos (Pastor Pascual, 2021) que repercuten en la práctica y elección de determinadas disciplinas (Guerrero, 2021) o que, entre otras, el embarazo se considere en los deportes de alto rendimiento una enfermedad de la deportista y en el deporte cotidiano todavía esté envuelto en importantes tabús que evidencian la desatención científica y social al respecto, son algunos de los muchos ejemplos que demuestran que el deporte no ha dejado de ser un espacio profundamente masculinizado (Begoña Marugán, 2019), diferenciando de manera jerarquizada categorías binarias (hombres y mujeres, competiciones femeninas y competiciones masculinas) y provocando la perpetuación de las limitaciones y discriminaciones de los cuerpos ${ }^{5}$.

Es por esto que, para hablar de los sistemas de organización de los equipos deportivos y cómo intervienen en las formas en las que sus agentes expresan sus emociones dentro de ellos, deberemos aproximarnos a lo que García García (2009) introdujo como los laboratorios de género. El deporte y los equipos deportivos, en tanto contextos herederos de esos espacios de masculinidad acomodados en una autoridad principalmente masculina (Hargreaves, 1993), no sólo serán todavía espacios perfectos para la exaltación de rasgos de género tradicionales (Pfister, 2010), además mostrarán formas muy características de reproducirlos, exponiendo férreas categorías binarias atravesadas por el poder de manera explícita, pero al mismo tiempo en convivencia con una sociedad en la que cada vez habría menos cabida para ello. Estos laboratorios de género pretenden aportar una nueva perspectiva de análisis, entendiendo el deporte, y en concreto los equipos deportivos, como espacios en los que la participación de las mujeres es 
creciente, no existiendo actualmente posibilidad de negar su presencia y reconociendo sus luchas en los últimos años, pero al mismo tiempo hostil y discriminatorio, con dinámicas de reproducción de esas performatividades hegemónicas (Butler, 2005) y bajo unas regulaciones heteronormativas que pivotan en una superposición de la autoridad masculina (Blanco García, 2021). El deporte femenino y las mujeres en el deporte forman parte de la actualidad, pero eso no quiere decir que su participación sea ni mucho menos inclusiva, lo que hace interesante atender a las regulaciones presentes en sus dinámicas cotidianas y coreografías de sus encuentros (Casado, $2014)^{6}$.

Dicho esto, se encuentran en las entrevistas realizadas relatos que sustentan esta normalización de las discriminaciones de género a lo largo de la carrera deportiva, por parte de hombres y mujeres y en roles de deportistas y entrenadores/as, sin importantes distinciones. Sin embargo, interesa destacar en concreto uno de los fenómenos que se han repetido en prácticamente todos los relatos de las mujeres, jugadoras y entrenadoras: las profecías autocumplidas (Sánchez García, 2020), siendo además sus relatos reforzados por sus compañeros. Bajo esta descripción, se ha encontrado en las mujeres, principalmente deportistas pero no sólo, una determinada forma de asumir y normalizar tradicionales estereotipos de género que limitan su capacidad deportiva: excesiva expresión emocional, miedo al descontrol emocional de las mujeres, pero también dificultades en el liderazgo y el ejercicio de su autoridad. Si bien se han repetido de maneras similares en todas las entrevistas, la forma de relatarlos por parte de las mujeres deja entrever una asunción de la pérdida de poder en el contexto deportivo, acompañado de un especial compromiso de esfuerzo y superación en el caso de las entrenadoras. Además, dirigiéndonos directamente a esos prejuicios y estereotipos ligados a las emociones, cabe destacar un particular miedo al descontrol emocional del equipo que es respondido por parte de los entrenadores con mayor liderazgo y autoridad.

Somos más susceptibles. Nos lo tomamos todo más a pecho. También, somos más capaces de dialogar, entonces creo que solventamos los problemas de mejor manera. Pero, creo que somos muy, como «¿qué has dicho?»Y nos lo tomamos mal. No sé. (Jugadora, 20 años).

Género, poder y liderazgo: la convivencia en los equipos deportivos

El mundo es comprensible, está inmediatamente dotado de sentido, porque el cuerpo, que, gracias a sus sentidos y su cerebro, tiene la capacidad de estar presente fuera de sí, en el mundo, y de ser impresionado y modificado de modo duradero por él, ha estado expuesto largo tiempo (desde su origen) a sus regularidades (Bourdieu, 1999: 180).

$\mathrm{Si}$ estos laboratorios de género ya vienen exponiendo una reproducción de las performatividades de género asociadas a formas tradicionales, estudiar las disposiciones del poder y sus juegos con los diferentes roles establecidos va a señalar directamente interpretaciones y exposiciones emocionales, no sin visibilizar al mismo tiempo importantes tensiones en sus regulaciones.

Los equipos deportivos serán grupos de personas no elegidas organizados en una férrea jerarquía vertical, donde el poder, la autoridad y el liderazgo van a ser imprescindibles para su convivencia (Messner, 1992). En ella, podrán sucederse dinámicas de control y obediencia que actualmente serían desproporcionadas en otros contextos, pero tienen cabida en el deporte a través de fuertes idealizaciones de ciertos valores sociales ligados al éxito y la disciplina (Bromberger, 1995). Estas disposiciones del poder serán encarnadas con una cierta docilidad (Foucault, 1997) que, aun pudiendo exponer resistencias, normalmente viajan entre fronteras que justifican tales dinámicas, tan características como valoradas, propias de los equipos deportivos. Tal y como se expone en la Figura 1, tomando especial relevancia los agentes implicados dentro del propio equipo, se encarna una distribución del poder en forma de pirámide inversa, con una pérdida de este poder según disminuyen las posiciones hasta la base.

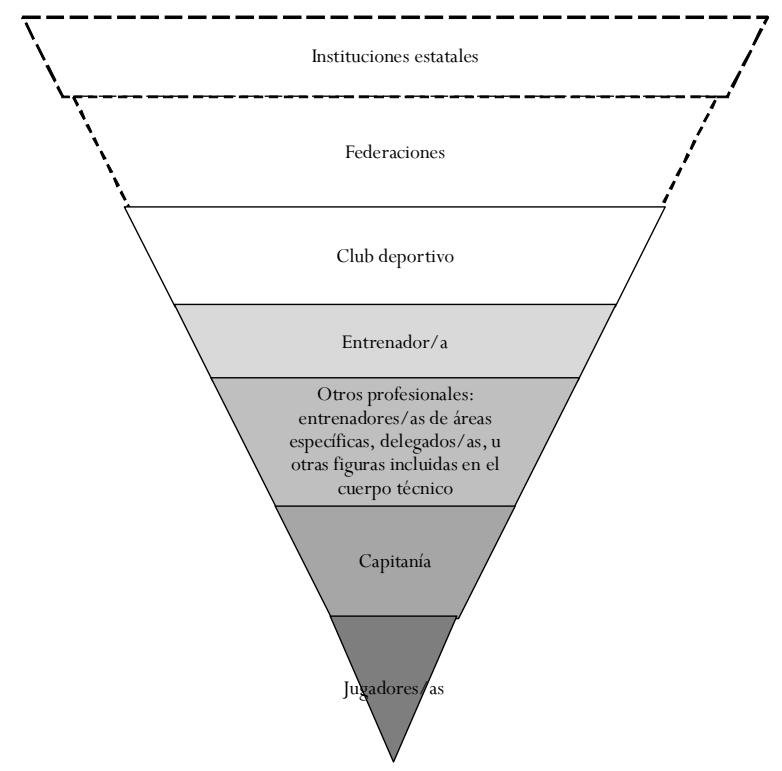

Figura 1. Pirámide representativa de la distribución del poder en un equipo deportivo. Nota: Esta pirámide representa la distribución del poder en los equipos deportivos, de forma invertida para exponer la concentración de poder en las posiciones más altas y la progresiva pérdida del mismo. Se trata de una elaboración propia a partir del análisis de las entrevistas realizadas en el estudio. 
El poder atraviesa de estas formas los cuerpos deporti$\operatorname{vos}^{7}$. Un poder que no recae en forma vertical, de manera unilateral y asienta los cuerpos sin posibilidad de resistencia, sino que más bien atraviesa estos cuerpos en múltiples direcciones, sin fronteras fijas, en juegos y tensiones que viajan desde los diferentes roles y las regulaciones heteronormativas en su encarnación, hacia el conjunto de valores y normas explícitas e implícitas que les llevan a interactuar bajo dinámicas reconocidas en el contexto. Así ha sido definido por todos los agentes entrevistados, que de una u otra forma han descrito estas formas de reparto del poder, dando toda la autoridad posible, en tanto capacidad de decisión, gestión del grupo, y similares, al entrenador/a, y encontrando en los y las deportistas la obediencia de tales órdenes.

P:Y, ¿quién debe mandar estas cosas? ¿Definir quién las hace?

R: Para mí es el entrenador. O sea, porque al final es... Así me lo tomo yo y toda la confianza como jugadora se la tengo que depositar a él. A mi equipo, pero a él para que lo dirija. Yo puedo estar de acuerdo con una jugada o no, comentar con él si me gusta o no. Pero no soy nunca quién para decir, si manda rojo no, hacemos azul porque me sale a mí de las narices. O sea, que si nos equivocamos todos, nos equivocamos, pero depositamos la confianza en quien al final tiene la última palabra (Jugadora, 25 años).

Este retazo de entrevista ratifica lo que acaba de explicarse, la autoridad indiscutible dentro del equipo será la del entrenador, con importantes idealizaciones ligadas al éxito en y de su exposición masculina normativa (García García, 2009; 2010). El rol de entrenador/a encarnará liderazgo, poder y disciplina, siendo responsable del control y estabilidad del equipo. Un rol masculinizado, con necesidades de reconocimiento masculino que viajarán hacia un liderazgo potencialmente autoritario, sobre todo en el caso de los hombres ${ }^{8}$.

P: ¿Te parece importante liderar tu vestuario?

R: Sí. Para mí, yo soy responsable de ser el líder de ese grupo de personas. Si tú no eres el líder lo va a ser otra persona, y si no es otra persona tú ya no controlas lo que hay en tu vestuario. Entonces, es súper importante ser el líder. Es verdad que tienes que tener ciertos apoyos dentro del vestuario que te vayan informando, que te apoyen en tus decisiones, pero el líder tiene que ser el primer entrenador (Entrenador, 34 años).

Como ya se ha mencionado, esta asunción del poder es universal en los diferentes roles del equipo, desde deportistas hasta entrenadores/as representan esa distribución del poder de la misma manera y asumen sus roles y responsabilidades. Sin embargo, cabe preguntarnos qué ocurrirá concretamente con el conjunto de deportistas, cómo encarnan su rol y cómo gestionan sus posiciones según estas representaciones, siendo la suya la posición más vulnerable.

Para empezar, es preciso recordar las profecías autocumplidas que han sido mencionadas anteriormente. Estas profecías autocumplidas viajan en diferentes direcciones y una de ellas tendrá mucho que ver con las formas de expresión emocional en la propia asunción del poder dentro del equipo. Se demandará una importante autoridad del entrenador para controlar aquellas situaciones que pueden ser susceptibles al descontrol, principalmente tratándose de equipos femeninos. A partir de aquí, los equipos se verán atravesados por una estricta homogeneidad que representará uno de los valores más importantes entre los y las deportistas. Tanto jugadores como jugadoras lo representarán de la misma manera, pero será entre las deportistas entre quienes más se señale, tanto entre las propias deportistas, como entre sus compañeros y entrenadores, ligado nuevamente a esos prejuicios y estereotipos emocionales. Así que, con esta homogeneidad del grupo, no sólo nos encontramos una exposición de valores deportivos, como pueden ser el compañerismo, la generosidad o la empatía entre compañeros/as, sino que viaja hacia una estricta igualdad que presenta dificultades en la interpelación y reconocimiento de cada deportista individualmente.

Pero, mientras que ninguna destaque o... ¿sabes? Si todo sigue como igual, es que cuando un grupo así es homogéneo y ninguna... pues lo típico (Jugadora, 25 años).

Cabe destacar que, a pesar de que se proclame esta homogeneidad, principalmente entre las jugadoras, pero no sólo, lo cierto es que no existen principios estáticos que la corroboren, ni tampoco puede ser efectiva en la práctica, creando de esta forma continuas tensiones en su convivencia y en la asunción de las disposiciones que se determinaban en la Figura $1^{9}$. Así pues, se demuestra que en estas disciplinas de equipo existirán ciertas resistencias hacia la diferenciación e interpelación de miembros de manera individual, lo que hace todavía más compleja la distribución de los poderes y la demanda de autoridad en la figura del/a entrenador/a. Y es que, se depositarán en ella todas estas responsabilidades de interpelación individual y, con ello, se potenciarán los juegos y encarnaciones de poder que ya están dispuestos de manera explícita en el propio equipo. Por ejemplo, una de las dinámicas más recurrentes de in- 
terpelación individual cedida a la figura de entrenador/ a será la definición de cada deportista y sus funciones dentro del equipo. Tal y como muestra el siguiente fragmento de entrevista, será el propio equipo quien, huyendo de las incomodidades que podrían surgir al definirse y diferenciarse de sus propios/as compañeros/as, deposita esta función al propio entrenador, esperando que su rol sea impuesto dentro de unos límites que no rompan con su inclusión en el núcleo de deportistas.

Pues mira, este año mi entrenador hizo una charla al principio del año y nos definió poco a poco, una a una. Nos conocía a algunas, a otras no. A mí me conoce porque me ha entrenado 5 o 6 años y dijo: «mirad, esta chica si queréis que rinda y que sea alegre, que rinda, que es un espectáculo, no sé qué, no sé qué...», en plan, lo típico que pone a todas un poco por las nubes (Jugadora, 24 años).

En este caso se expone en forma de demanda desde el rol de deportista, pero lo cierto es que si se enfoca desde el rol de entrenador/a se considera además dentro de lógicas de definición y control del propio equipo. Además, destacar que, a pesar de que estas lógicas homogéneas atraviesan de igual forma los equipos femeninos y masculinos, estamos viendo que resulta un mayor reclamo entre las deportistas, algo que tendrá mucho que ver con los intercambios producidos entre entrenador y jugadora, tal y como se verá a continuación. De momento, cabe preguntarnos si esta capacidad de interpelación y reconocimiento otorgada exclusivamente a la figura de entrenador/a es primero una demanda de los y las deportistas o una dinámica asentada en el contexto que provoca la interiorización de tales definiciones y necesidades homogéneas. Pues bien, lo que demuestran los relatos de los entrenadores tiende hacia esa imposibilidad de construir un equipo efectivamente homogéneo, pero en los mismos intentos de reforzar una asunción del rol a través de este tipo de ritualizaciones de definición y al mismo tiempo asentamiento del poder.

Creo, y me parece lo más complicado, que lo más importante es que todo el mundo acepte su rol. Hay roles que molan, el que necesitas que meta puntos, rebotear, jugar 30 minutos... Y otros roles que molan menos, que necesitas que defienda o ir al rebote pero que cuando lo cojas, sueltes el balón... Si, como entrenador, consigues que las personas entiendan su rol, lo acepten y sigan, da resultados, tienes muchísimo ganado (Entrenador, 36 años).

Tal y como demuestra este retazo, aun cuando no existe una demanda explícita, se considera una responsabilidad adherida al rol de entrenador manejar el grupo, y en ello aparece la definición de los roles de cada jugador/a. Se conforman perfiles concretos y se categoriza a cada miembro en ellos, como si se tratase de un puzzle rígido que debe de cualquier manera encajar.

A partir de aquí, recuperando las formas de organización de los equipos atravesados por estas disposiciones, tal y como destacó Messner (1992), si algo queda revelado es que estas formas responderán a unas estructuras de disciplina y obediencia que señalarán precisamente la necesidad de que exista un poder concentrado en una figura entendida como superior, el/la entrenador/a, y un conjunto de miembros prácticamente limitados a seguir las indicaciones. Ahora bien, una vez más, pareciera que las estructuras son rígidas, cuando realmente pueden existir resistencias, y así lo demuestran las desviaciones existentes en el rugby, que manteniendo las mismas concepciones del poder y la responsabilidad de los diferentes roles, expone prácticas cotidianas diferentes, con menos tensión dentro del núcleo de deportistas. Aun así, existe algo que no podemos negar: la fuerte jerarquización del equipo en cualquiera de las disciplinas; y es que desde los primeros contactos dentro de un equipo, en la infancia, se expone una jerarquización en la que uno de los principales valores de aprendizaje será la disciplina, enfocada en la obediencia a figuras entendidas como superiores. Aprendizajes que conviven en muchos de nuestros contextos primarios, como la familia, y segundarios, como la escuela, siendo reforzados en el contexto deportivo como un espacio de especial idealización de estos valores de obediencia y respeto (Messner, 1992). Esto provoca la interiorización de tales disposiciones y asienta sus estructuras y, aunque no sea la única opción, sí existen ciertas tendencias que confirman que el contexto deportivo, y en concreto los equipos deportivos, se configuran en espacios donde se reproducen sistemas de obediencia y disciplina exacerbados a lo largo de la vida, con un reconocimiento jerárquico poco habitual en otros ámbitos similares ${ }^{10}$. Desde aquí, se registran ciertas tendencias que pueden ir desde estas asentadas exposiciones de la autoridad y el mandato del equipo y la toma de decisiones, hasta prácticas que incurren en elevados tonos de voz e incluso insultos o descalificaciones que pueden propiciarse de entrenador/a a deportista y que muchas veces se normalizan, otras incluso se justifican ${ }^{11}$.

Todo ello conduce a cuestionar la base que sustenta estas prácticas y sistemas. Es decir, ¿por qué son norma- 
lizadas ciertas prácticas en estos contextos deportivos? ¿Dónde encuentran, más allá de las propias estructuras, el sustento para su aprobación e incluso normalización en nuestra sociedad actual? En numerosas ocasiones se ha buscado la explicación en la base competitiva del deporte y al mismo tiempo se hace referencia a la implicación emocional que conlleva esta competitividad. Todo ello, en un contexto masculinizado con una potencial exposición de ciertos rasgos de una masculinidad normativa (García García, 2009) que efectivamente influyen en sus dinámicas cotidianas. Es decir, una especie de contradicción entre la liberación emocional (masculinizada), evasión y desconexión de las exigencias cotidianas que se vienen destacando desde los inicios del deporte moderno (Elias, 1986), hacia una progresiva racionalización de estas prácticas deportivas, siendo cada vez más la exigencia de control y el rechazo a ciertas explosiones emocionales, pero al mismo tiempo manteniendo una residual justificación que nace, en cierta forma, de la asentada masculinización del contexto ${ }^{12}$.

\section{Expresiones emocionales y aparición de espa- cios de vulnerabilidad}

El interés por los estudios de las emociones en el deporte es cada vez mayor (Bermúdez Torres y SaenzLópez, 2019). Por eso, es en este momento en el que las emociones entran en juego y hacen imprescindible su estudio para comprender estos juegos cotidianos, apareciendo en forma de sustento de las estructuras jerarquizadas y sus tensiones de disciplina y poder. Tal y como se venía introduciendo, esas prácticas exacerbadas que pueden llegar a experimentarse en estos equipos deportivos, fruto del aprendizaje y normalización de los sistemas, necesitan encontrar un anclaje que favorezca su justificación y no rompa sus ritualizaciones. Este anclaje puede residir en las sensibilidades y emociones intensas que caracterizan la convivencia en los equipos, en tanto contextos de gran emocionalidad (Elias, 1986), siendo grupos de personas que conviven en una característica intensidad de sus experiencias, mediadas en todo momento por la sensibilidad a la que se exponen sus miembros y la fuerza que se percibe en sus vínculos.

Cuando tú tienes una apertura emocional muy grande, es un contexto en el cual estás mucho más abierto a tener relaciones personales con otras personas. Por ejemplo, en un campo de rugby, después de 80 minutos de golpes, placajes, sudor, de caerte, levantarte, de placar con tu compañero, de la adrenalina que tiene el correr con un balón y que tengas tres tías detrás que te quieren placar, en ese momento das el pase a un compañero y hace un ensayo. Toda esa adrenalina, todas esas endorfinas, todas esas emociones que se sienten en un partido, ante una lucha, ante una victoria, ante un tal... Son situaciones en las cuales nos abrimos emocionalmente, porque, bueno, es lo que tiene el deporte, ¿no? Está estudiado (Jugadora, 30 años).

Esta «apertura emocional» relatada en esta entrevista de una jugadora define perfectamente las sensaciones que han sido referidas en una y otra entrevista, prácticamente de las mismas formas. Ahora bien, es especialmente interesante destacar esta sensibilidad en el caso de los y las deportistas porque, a pesar de que es reconocido de igual manera por todos los miembros de los equipos, lo cierto es que es en los roles de deportistas en los que más importancia toman las afectividades y vínculos con los/as compañeros/as. Desde aquí, cabe primero destacar la implicación de los cuerpos y el contacto corporal en esta conexión emocional, pues el sustento de las emociones sobre el que voy a dialogar en las siguientes páginas, las formas características en las que se gestionan las emociones, los vínculos y las dinámicas afectivas que aparecerán en estos equipos deportivos que implican contacto con el rival en su práctica, parten precisamente de entender eso, el contacto. El contacto de los cuerpos al chocarse, abrazarse, empujarse, ayudarse a levantar, etc., esos contactos corporales trazan el camino en el que discurren las emociones en estos equipos. Por ello, esa adrenalina, esas emociones que resume el retazo de la entrevista anterior, no sólo hablan de las emociones en el deporte, como sobre todo de las emociones a través del contacto corporal en estas disciplinas deportivas.

Tú, quedas con gente de anteriores equipos y no es lo mismo que si quedas con gente de fuera del baloncesto. Recuerdas las cosas que hiciste, de victorias, derrotas, fiestas, cosas del vestuario, lesiones... Ya sean cosas buenas o malas, no sé, lo recuerdas con una sonrisa en la cara. Me parece algo más difícil, lograr esa unión que la que puedes hacer fuera de la pista con cualquier otra gente. Entonces, por eso yo lo valoro más, las amistades que haces de verdad dentro de la cancha que las de fuera, porque me parece algo mucho más complicado (Jugador, 21 años).

Inevitablemente, la intensidad de las emociones y los vínculos, incluso de la amistad, se comparan entre los/as compañeros/as de equipo y quienes forman parte de otros espacios, como pueden ser la escuela o el trabajo. Se comparan para señalar precisamente la intensidad y cercanía lograda en los equipos, la conexión 
que puede alcanzarse entre compañeros/as principalmente, pero también con el cuerpo técnico a lo largo de una temporada cargada de experiencias intensas. Es decir, lo importante en estos deportes no serán sólo las sensaciones de evasión y/o desconexión de nuestras exigencias cotidianas, como sobre todo las implicaciones y reciprocidades que esperamos de quienes forman parte de nuestro equipo. En este sentido, Messner (1992) comprobó cómo muchos deportistas se referían a sus entrenadores como «dioses» o «padres» y relacionó estas idealizaciones con las continuas referencias a sus equipos como «segundas familias», siempre en términos positivos y agradables. A continuación, estudió cómo estas referencias en realidad podrían enmascarar un importante tejido de jerarquización, control y disciplina. Es aquí donde se encuentra la conexión entre las tensiones mencionadas en el punto anterior y esta perspectiva emocional con la que nos acercamos a los equipos deportivos. Y es que, precisamente esta intensidad emocional que es expuesta en todas las entrevistas como beneficiosa para el propio equipo y cada uno de sus integrantes, realmente constituye la base de sostenimiento del sistema y sus tensiones. De hecho, esta forma relevante de relatarlo desde la perspectiva de los y las deportistas, siendo la base de aquella pirámide descrita en la Figura 1, dice mucho de la configuración del sistema, mediado por idealizaciones y aprendizajes de la autoridad y la obediencia. Las emociones se hacen intensas, al tiempo que la idealización de la figura del/a entrenador/a se refuerza, no sólo asumiendo su autoridad, sino además situándola como objeto de deseo, especialmente en los equipos femeninos debido a la heteronormatividad del propio sistema (Blanco García, 2021). El resultado es una justificación del sistema jerarquizado, la disciplina y el control autoritario a través de la idealización de la sensibilidad e intensidad emocional del contexto, así como otros valores socialmente idealizados en el deporte.

Con todo ello, podemos determinar que la especial sensibilidad y la cercanía de los equipos deportivos sirven como ese sustento al estricto sistema jerárquico, porque actúan en justificación y respaldo de aquellas situaciones que efectivamente resultarían fuera del espectro actual. Es decir, la intensidad emocional experimentada a través de la práctica deportiva y la convivencia con el grupo, especialmente en el caso de los y las deportistas, hacen la pertenencia al grupo valiosa y única, incomparable a otros contextos cotidianos. Por otra parte, la idealización de esa «segunda familia» provoca la encarnación de unos roles atravesados por tensiones de poder que vuelven a idealizar la figura del/a entrenador/a, siempre bajo una masculinización tradicional del rol, provocando en cierta forma un juego perverso. $\mathrm{Y}$ es que, pudiera parecer que es esta «segunda familia» la que demanda las formas de gestión autoritarias y no al revés. Es decir, las dinámicas y juegos de poder y estricto disciplinamiento que han sido definidos anteriormente, ¿surgen como respuesta a las necesidades emocionales de los equipos? ¿O son precisamente las estructuras y sistemas los que hacen necesarias las idealizaciones de una metafórica «segunda familia» para perpetuar sus rituales ${ }^{13}$ ? Caer en la trampa de que puedan ser las necesidades emocionales la razón de la posterior construcción de tales sistemas sería contribuir a la perpetuación de la justificación de estas prácticas desproporcionadas y roles potencialmente autoritarios en el deporte. Y es que, el hecho de idealizar a la figura a la que se le otorga poder, reforzando este reparto explícito del mismo en tales estructuras, vuelca en ella cantidad de expectativas, tanto en la propia gestión del grupo, como en la interpelación de los diferentes miembros de manera individual. Este tema no lo podremos abordar en este momento, pero merece la pena mencionarlo ante la pérdida de control que supone para quienes están asumiendo estas circunstancias desde la docilidad (Foucault, 1997), sobre todo quienes aparecen en la base de esa pirámide mostrada en la Figura 1. El sistema se visualiza como frágil, ante estas contradicciones entre las propias estructuras y el sustento emocional que no encuentra su propio anclaje como base.

Así pues, las reflexiones continuarían hacia la visibilización de las vulnerabilidades que pueden despertarse en las relaciones que se establecen dentro de estos equipos deportivos, sobre todo entre cuerpo técnico y deportistas, por las profundas idealizaciones y exposiciones de autoridad que pueden percibirse difuminadas ante las sensibilidades y expresiones emocionales.

\section{Conclusiones}

Un primer acercamiento a las emociones en los equipos deportivos debe partir de la descripción del contexto, teniendo en cuenta todas sus características y muy especialmente aquellas que influyen de manera concreta en las formas de reproducir y reinterpretar sus interacciones. Es por lo que analizar las formas en las que se expone la autoridad y el poder en los equipos deportivos ha sido uno de los puntos de partida imprescindibles para este estudio. 
En este sentido, se ha abierto en este artículo un acercamiento a los equipos deportivos como laboratorios de género en un intento de visibilizar la hostilidad y discriminación del propio contexto, teniendo en cuenta las regulaciones heteronormativas que se reproducen día a día y gestionan sus estructuras bajo una estricta masculinización del contexto. Una hostilidad que no pivota en prohibiciones explícitas, sino que se esconde en la normalización, pues la eliminación de estas prohibiciones que sí existieron no hace mucho tiempo nunca ha significado un paso real hacia la inclusión de las mujeres en el deporte, mucho menos de las diversidades. El deporte ha sido siempre un contexto masculinizado, creado por y para hombres (Sabo y Panepinto, 1990; Díez Mintegui, 2006) y las únicas posibilidades que ha abierto han viajado hacia una estructuración heteronormativa que reconoce categorías binarias en las que una está superpuesta a la otra. Las mujeres nunca han tenido la oportunidad de preguntarse si el diseño de este deporte moderno cumple realmente sus expectativas, ni siquiera si cubre sus necesidades, puesto que sus posibilidades han ido siempre ligadas a la adaptación de un espacio que no se ha diseñado para ellas, no se ha hecho suyo, simplemente se ha abierto a su participación.

Desde aquí es desde donde se deben estudiar las estructuras de los equipos deportivos y, con ello, valorar las expresiones emocionales, afectividades y vínculos en sus convivencias. Se ha demostrado una exposición jerárquica que presenta sistemas de disciplinamiento y obediencia férreos naturalizados a lo largo de la vida. Estos sistemas abogan por una idealización de una masculinidad normativa en la figura de entrenador/a que conlleva múltiples exigencias para quien lo encarna, pero también idealizaciones para quienes asumen y demandan su liderazgo. Se encuentran entonces representaciones autoritarias que pueden llegar a escapar de consideraciones actuales, principalmente a través de una justificación de ciertas prácticas exacerbadas que se relacionan directamente con las especiales sensibilidades del contexto en dos líneas de influencia. En primer lugar, en la justificación de estas prácticas masculinizadas, totalmente desproporcionadas en otros contextos; pero sobre todo en la utilización de estas sensibilidades como sustento a las propias contradicciones del sistema. En esta segunda línea se ha incidido en el artículo, con una importante reflexión en el camino; y es que, cabe el debate en torno a las diferentes disciplinas estudiadas, entendiendo que, si bien las bases del propio deporte moderno han asumido estas consideraciones, sus pro- pios pilares deben reformularse para romper con muchas de las estructuras que se perpetúan. Esto, sin desmerecer las resistencias y buenas prácticas que han aparecido en disciplinas como el rugby, que abogan por nuevas dinámicas que, aun bajo unos mismos parámetros, escapan de la centralización de la autoridad en una figura y aportan mayor independencia y capacidad al propio núcleo de deportistas. Eso sí, aun con todo, persiste de manera universal la idealización de un poder que se reconoce en la misma estructura piramidal.

Con todo ello, llegamos al papel que juegan las emociones en la mediación del entorno, siendo realmente la base para entender las interacciones que en estos sistemas se producen y hacia la visibilización de ciertas vulnerabilidades de los encuentros que no hemos llegado a señalar en este artículo. Y es que, si las especiales sensibilidades del contexto son utilizadas como la justificación de ciertas prácticas y las afectividades y la creación de vínculo las sustentan al mismo tiempo, ya no sólo en una justificación, sino en una especie de validación en forma de balanza, ¿cómo se gestionarán las relaciones en estos equipos deportivos? Es decir, ¿cómo se señalarán las fragilidades de sus contradicciones, las rupturas en esas «segundas familias», sin romper la esencia del espacio? La dificultad de enfrentar estas cuestiones suele conducir una huida hacia la potenciación de la idealización de estas sensibilidades y afectividades, siempre mediadas por/en estos laboratorios de género que podrían provocar la invisibilización de ciertas vulnerabilidades en las relaciones dentro de los equipos deportivos, especialmente femeninos.

Para terminar, tal y como se introducía en la metodología, existe un interés especial en esta investigación en focalizar ciertos espacios de interacción que pueden resultar vulnerables, donde estas prácticas exacerbadas que se han señalado pueden favorecer la normalización de dinámicas de acoso sexual que serían inmediatamente desaprobadas en otros contextos sociales. En este artículo, refiriéndonos a uno de los capítulos del estudio en su conjunto, se han querido focalizar las formas de organización y estructuras dentro de los equipos deportivos, así como las expresiones y mediaciones emocionales. Se ha demostrado que efectivamente existen dinámicas que pueden favorecer tal vulnerabilidad en el contexto, erguidas sobre una heteronormatividad que potencia la idealización de la figura masculinizada del entrenador y ciertas capacidades adheridas a ella, abogando además por una creciente autoridad y liderazgo concentrados en su rol. Con todo ello, queda expuesta la razón de este urgente acercamiento a las dinámicas 
afectivas en estos espacios, continuando hacia esas dinámicas sexo-afectivas que partirán de estos análisis para concretar cómo inciden tales prácticas en las relaciones entre entrenadores jóvenes y adultos y jugadoras jóvenes, en un intento de visibilizar y concienciar sobre estas vulnerabilidades y los abismos hacia los que pueden conducir sus huidas.

\section{Referencias}

Alfaro, Élida (2008). Mujer joven y deporte. Revista de Estudios de Juventud. $\mathrm{n}^{\circ} 83$, p. 119-141. INJUVE.

Alonso, Luis Enrique (1994). Sujeto y discurso: el lugar de la entrevista abierta en las prácticas de la sociología cualitativa. En: Delgado, José Manuel Y Gutiérrez, Juan (coords.). Métodos y técnicas cualitativas de investigación en Ciencias Sociales. Madrid: Síntesis.

Alonso, Luis Enrique (1998). La mirada cualitativa en sociología. Madrid: Fundamentos.

Amorós, Celia (1991). Hacia una crítica de la razón patriarcal (pensamiento crítico, pensamiento utópico). Anthropos. Editorial del Hombre.

Bericat Alastuey, Eduardo (2000). La sociología de la emoción y la emoción en la sociología. Papers. Revista de Sociología, pp. 145-176.

Bermudez Torres, Carlos; Saenz-López, Pedro (2019). Emociones en Educación Física. Una revisión bibliográfica (2015-2017). Retos, n 36, pp. 597-603.

Blanco García, Marta Eulalia (2021). Deporte, cuerpo y género: los equipos deportivos como espacios de vulnerabilidad. Memoria para optar al grado de Doctor. Universidad Complutense de Madrid.

Böhm, Arno (1993). ¿Deportistas o mujeres? Martina Navratilova lo consiguió. Geu Emakumeok, n¹6, pp. 33-34.

Bourdieu, Pierre (1993). Deporte y clase social. En: Brohm, J. (1993). Materiales de sociología del deporte. La Piqueta.

Bourdieu, Pierre (1999). El conocimiento por cuerpos. En: Bourdieu, Pierre. Meditaciones pascalianas: 171-214. Barcelona: Anagrama.

Brackenridge, Celia (2003). Dangerous sports? Risk, responsibility and sex offending in sport. Journal of Sexual Agression, 9 (1): 3-12.

Bromberger, Christian H. (1995). Le match de football. Éditions de la Maison des Sciences de L'Homme.

Butler, Judith (2005). Regulaciones de género. La ventana, $n^{\text {o } 23, p p . ~ 7-35 . ~ T r a d u c c i o ́ n ~ d e ~ M o i s e ́ s ~ S i l v a . ~}$

De Lauretis, Teresa (1992). Semiótica y experiencia. En: Alicia ya no. Valencia: Cátedra: 251-294.
Díez Mintegui, Carmen (1996). Deporte y construcción de las relaciones de género. Gazeta de Antropología, 1996, 12 , artículo 10.

Díez Mintegui, Carmen (2006). Juventud y deporte. Una propuesta de cambio en la organización tradicional de la actividad deportiva. Revista de Dialectología y Tradiciones Populares, vol. LXI, n², pp. 129-144.

Elias, Norbert (1986). Deporte y violencia. En: Wright Mills, C. et all. (ed). Materiales de sociología crítica. Genealogía del poder. Madrid, España.

Esteban, Mari Luz (2013). Antropología del cuerpo. Género, itinerarios corporales, identidad y cambio. Edicions Bellaterra, S.L.

Fasting, Kari; Brackenridge, Celia; Sudgot-Borgen, J. (2003). Experiences of sexual harassment and abuse among Norwegian elite female athletes and non athletes. Research Quarterly for Exercise and Sport, 74 (1): 84-97.

Fausto Sterling, Anne (2006). Cuerpos sexuados. La política de género y la construcción de la sexualidad. Traducido por Ambrosio García Leal. Editorial Melusina.

Finkel, L.; Parra, P. y Baer, A. (2008). La entrevista abierta en investigación social: trayectorias profesionales de ex deportistas de élite. En: Gordo A. y Serrano, A. (coords.). Estrategias y prácticas cualitativas de investigación social. Editorial Pearson.

Gallardo Peña, Manuel Antonio; Domíngez Escribano, Marta; González González de Mesa, Carmen (2018). Inteligencia emocional y conducta agresiva en el deporte ¿Puede inferir la modalidad deportiva y las horas de entrenamiento? Retos, $\mathrm{n}^{\circ} 35$, pp. 176-180.

García García, Antonio Agustín (2009). Modelos de identidad masculina: Representaciones y encarnaciones de la virilidad en España(1960 2000). Memoria deTesis Doctoral. Universidad Complutense de Madrid.

García García, Antonio Agustín (2010). Exponiendo hombría. Los circuitos de la hipermasculinidad en la configuración de prácticas sexistas entre varones jóvenes. Revista de estudios de juventud, $n^{\circ} 89$, p. 59-78.

García García, José (2015). El origen del deporte femenino en España. Full Color Print Color.

Goffman, Erving (1979). Relaciones en público. Microestudios del orden público. Madrid: Alianza.

Grosz, Elizabeth (1994). Volatile Bodies.Towards a Corporeal Feminism. Bloomington and Indiannapolis: Indiana UP: 3-61.

Guerrero, David (2021). Corres como una niña. Editorial Dos Bigotes.

Haraway, Donna (1988). Situated Knowledges: The Science Question in Feminism and the Privilege of Partial Perspective. Feminist Studies, vol. 14, n 3, pp. 575-599. 
Hargreaves, John (1993). Promesa y problemas en el ocio y los deportes femeninos. En: Barbero, J. I. (ed.). Materiales de sociología del deporte. P. 109-132. La Piqueta.

Iturbide, Luis María; Elosua, Paula (2012). Percepción del "fair play» en deportistas infantiles y cadetes. Revista de Psicología del Deporte, $n^{\circ} 21$ (2). P. 253-259.

Messner, Michael A. (1992). Power at play. Sports and the problem of masculinity. B. Press.

Ministerio de Educación, Cultura y Deporte (2015). Encuesta de hábitos deportivos en España. Secretaría General Técnica. Subdirección General de Documentación y Publicaciones. Gobierno de España.

Pastor Pascual, Ana (2021). Chandaleras. Masculinidad femenina vs feminidad obligatoria en el deporte. Piedra papel libros.

Pfister, Gertrud (2010). Women in sport - gender relations and future perspective. Sport in Society, Vol. 13, No. 2. P. 234-248.

Sabido Ramos, Olga (2010). El «orden de la interacción» y el «orden de las disposiciones». Dos niveles analíticos para el abordaje del ámbito corpóreo-afectivo. Revista Latinoamericana de Estudios sobre cuerpos, emociones y sociedad, vol. 3, pp. 6-17.

Sabo, Donald F. y Panepinto, John (1990). Football ritual and the social reproduction of masculinity. En: Messner, Michael A. y Sabo, Donald F. (1990). Sport, men, and the gender order. Kinetics Books.

Sainz de Baranda, Clara (2013). Mujeres y deporte en los medios de comunicación. Estudio de la prensa deportiva española (1979 2010). Tesis Doctoral. Universidad Carlos III de Madrid.

Sánchez García, Raúl (2020). Las cuatro heridas del deporte moderno. Colección libros del borde. Piedra Papel Libros.

Sonlleva, M., Martínez, A., \& Monjas, R. (2018). Los procesos de evaluación y sus consecuencias. Análisis de las experiencias del profesorado de Educación Física. Estudios Pedagógicos, 44(2), 329-351.

Subdirección General de Estadísticas y Estudios. (2019). Anuario de Estadísticas Deportivas 2019. Ministerio de Educación, Cultura y Deporte. Gobierno de España.

Vicente Olmo, Ana (2015). Representaciones y prácticas del amor entre la juventud española. Memoria para optar al Grado de Doctor. Universidad Complutense de Madrid.

\section{(Footnotes)}

${ }^{1}$ El hecho de valorar este contacto en la práctica nos conduce a tres disciplinas escogidas según similitudes y diferencias en su trayectoria y estado actual, así como el interés que suscita su comparación: baloncesto, fútbol y rugby. En las tres disciplinas se encuentran características concretas en cuanto al contacto corporal, la cercanía y el tráfico de emociones construido desde el contacto de los cuerpos, más allá de cuestiones fisiológicas. Además, estos deportes que implican contacto suelen implicar capacidades tradicionalmente masculinizadas (Bourdieu, 1993).

${ }^{2}$ La investigación es «Deporte, cuerpo y género: los equipos deportivos como espacios de vulnerabilidad». Memoria para optar al grado de Doctor (Blanco García, 2021).

${ }^{3}$ Remontándonos a los inicios del deporte moderno, a pesar de que nunca se ha podido negar la existencia de mujeres atletas, encontramos una prohibición explícita de su participación en competiciones oficiales. Por ejemplo, no fue permitida su participación en los I Juegos Olímpicos del Deporte Moderno (1896), de los que ya son conocidas las declaraciones de Pierre de Courbetin: «no es digno ni elegante que las mujeres participen en el deporte, resulta antiestético y carece de interés» (Alfaro, 2008: 129). Esta rotunda prohibición no duró demasiado, pues a partir de los II J.J.O.O. (1900) fueron progresivamente ampliándose las disciplinas en las que se permitía competir a las mujeres, acompañándose además de una creciente popularidad de la práctica deportiva en todos los países europeos que hizo incuestionable que las mujeres pudieran, igual que toda la población, practicar deporte.

${ }^{4}$ En España, en un impulso influido por otros progresos, principalmente del ámbito educativo, la participación de las mujeres en competiciones y actividades deportivas logró calar en la sociedad en el s. XX, siempre y cuando sus actividades deportivas fueran ligadas a una mejora de las condiciones biológicas relacionadas con la maternidad, en la preparación para el embarazo y la resistencia al parto, rechazando cualquiera que consideraran que pudiera ser contraria a estas y/o conllevar una masculinización o excesiva competitividad para su género (García García, 2015: 29-30).

${ }^{5}$ En coherencia con los planteamientos que se sucederán en cuanto a las emociones y dinámicas afectivas en los equipos deportivos, nos acercaremos a los cuerpos tomando la dualidad mente y cuerpo en conexión a través de desplazamientos que juegan entre lo interior y lo exterior, lo individual y lo social, lo consciente y lo inconsciente, de un lado a otro, en ambos sentidos (Grosz, 1994). En definitiva, los cuerpos como medios activos, como fronteras de mediación en la interpretación de 
sus realidades (Sabido Ramos, 2010: 11).

${ }^{6}$ La encuesta de hábitos deportivos en España (2015) muestra datos que relacionan una menor práctica deportiva de las mujeres, diferencias en las finalidades deportivas según el género o incluso diferencias en las ocupaciones de los espacios para practicar deporte. Otros estudios también han demostrado una menor representación de las mujeres en los medios de comunicación (Sainz de Baranda, 2013) y diferencias en la formación deportiva según el género (Anuario de Estadísticas Deportivas, 2019). Lo que se propone en este artículo es un acercamiento cualitativo a estas desigualdades, poniendo el foco en esas dinámicas afectivas y las vulnerabilidades que tienen su raíz en estas bases discriminatorias.

${ }^{7} \mathrm{Al}$ hablar de estos cuerpos deportivos no quiero en ningún caso limitarme a los cuerpos que realizan deporte, como sí a todos aquellos que conviven en el deporte, en los equipos deportivos. Es decir, aquellos que experimentan el contexto y conviven día a día en él, sea cual sea su rol dentro del equipo deportivo.

${ }^{8} \mathrm{Si}$ bien la autoridad de la figura de entrenador/a es reconocida por todos los agentes y la responsabilidad encarnada en el puesto presenta importantes similitudes entre entrenadoras y entrenadores, lo cierto es que las prácticas relatadas por mujeres y hombres son radicalmente diferentes. Los entrenadores presentarán, en estos casos, una necesidad de reconocimiento explícito de su autoridad por parte de los y las deportistas, debiendo reconocerse su rol en la dirección del equipo. Por su parte, las entrenadoras no sólo presentan dinámicas más participativas, sino que además rechazan la necesidad de verse explícitamente reconocidas como líderes del equipo.

${ }^{9}$ Sobre estos requisitos de homogeneidad del núcleo de deportistas, cabe destacar una especial atención en el baloncesto. Y es que, mientras que principalmente el rugby, pero también el fútbol, al presentar posiciones muy diferentes en el campo, con misiones y labores diferentes, pueden propiciar una menor comparación entre deportistas, el baloncesto presenta elementos de comparación en todas sus posiciones (número de puntos anotados, valoración cuantitativa de cada deportista comparable entre posiciones, etc.). Esta podría ser una de las razones por las que se ha observado una potenciación de esta homogeneidad en el baloncesto, ya no sólo en cuanto al trato entre deportistas, sino además al rol percibido en el terreno deportivo y su peso en el equipo.
${ }^{10} \mathrm{Tal}$ y como se ha introducido, resulta interesante en este aspecto atender a las similitudes y diferencias entre las diferentes disciplinas. Es decir, si bien todas las personas entrevistadas relatan una similar respuesta ante las estructuras de organización jerárquicas y la autoridad de la figura del/a entrenador/a, lo cierto es que las dinámicas y prácticas propias de cada disciplina presentan diferentes tendencias. Por ejemplo, mientras que en el mismo reglamento de rugby se evidencia una pérdida de autoridad de la figura de entrenador/a en los partidos, cediendo responsabilidad a la capitanía del equipo, en el reglamento de baloncesto se registran varios artículos que reafirman la autoridad de esta figura de entrenador/a.

${ }^{11}$ En las entrevistas, muchos agentes relataban experiencias propias o cercanas en las que se habían sucedido situaciones de esta índole, desde gritos y elevados tonos de voz en entrenamientos y partidos, pasando por insultos e incluso zarandeos a jugadoras. Cabe destacar que, aunque eran diferentes las personas que los relataban, de diferentes disciplinas, edades y géneros, todos los relatos señalaban estas experiencias como anecdóticas. También es interesante recalcar que ninguna de estas experiencias fue relatada como situaciones especialmente violentas que dieran pie a rupturas dentro del equipo e incluso del club o la federación.

${ }^{12}$ Como ejemplo, una patada a una botella, un grito e incluso un insulto, no se juzgarán socialmente de la misma forma en un contexto deportivo que en otros, ni siquiera se juzgarán de la misma forma según el género de quien lo protagoniza. Sobre estas reflexiones en torno a la agresividad en el deporte, existen estudios interesantes, como la publicación de Gallardo Peña et. al. (2018). Por otra parte, cabe destacar que los códigos de conducta, el conocido fair-play, incide cada día más en este tipo de violencias y agresiones (Iturbide y Elousa, 2012).

${ }^{13}$ Abordo los rituales y ritualizaciones desde las experiencias encarnadas a través de las convivencias cotidianas, propuestas por Goffman (1979; 1991; 2006) 